\title{
Tratamiento quirúrgico de las fractura de pared posterior de acetábulo. Resultados a corto y mediano plazo
}

\section{Surgical Treatment in Acetabular Posterior Wall Fractures. Short and Medium-Term Results}

\author{
Ignacio E. Villalón ${ }^{1,4}$ Jaime Cancino ${ }^{2 ®}$ Óscar Bravo ${ }^{3}$ Cristóbal Vigueras $^{3} \quad$ Julio Piriz $^{4}$ \\ Javier del Río ${ }^{1,5}$
}

${ }^{1}$ Equipo cadera y pelvis Hospital Clínico Mutual de Seguridad, Región

Metropolitana, Chile

2 Médico cirujano Universidad del Desarrollo, Santiago, Chile

3 Residente de Traumatología y Ortopedia, Universidad de Valparaíso, Chile

${ }^{4}$ Equipo cadera y pelvis Clínica Alemana de Santiago, Chile

${ }^{5}$ Centro de Cadera Clinica Las Condes, Región Metropolitana, Chile

Address for correspondence Ignacio E. Villalón, MD, Equipo cadera y pelvis Hospital Clínico Mutual de Seguridad, Región Metropolitana, Chile (e-mail: ignaciovillalonm@gmail.com).

Rev Chil Ortop Traumatol 2020;61:40-46.

\begin{abstract}
Resumen
Palabras clave

- Fractura acetábulo

- pared posterior

- reducción y osteosíntesis

Objetivo Evaluar los resultados radiológicos a corto y mediano plazo de pacientes operados de fractura de pared posterior de acetábulo, describir la morfología de la fractura, presencia de lesiones asociadas y la necesidad de artroplastia total de cadera (ATC) durante la evolución.

Material y métodos Estudio retrospectivo-descriptivo de una serie consecutiva de 40 pacientes operados entre julio de 2012 y julio de 2017 por fractura de pared posterior de acetábulo. Treinta y siete hombres y tres mujeres con una edad promedio de 41 años fueron operados (mediana de seguimiento 17,7 meses). Se registró el mecanismo del accidente y presencia de lesiones asociadas. Se registraron el número de fragmentos que presentaba la fractura, presencia de impactación marginal, compromiso de la cabeza femoral, presencia de fragmentos intra-articulares y complicaciones postoperatorias. Para el análisis estadístico se utilizó el test de Wilcoxon.

Resultados Todos los casos fueron secundarios a un accidente de alta energía. Un 70\% de los pacientes presentó luxación posterior. La mayoría de los pacientes presentó una fractura entre 2 y 5 fragmentos (70\%), 47,5\% de los pacientes presentó impactación marginal, $37,5 \%$ compromiso de la cabeza femoral en zona de carga, $30 \%$ fragmentos intra-articulares. La complicación que más frecuente se observó fue la artrosis posttraumática la cual se presentó en un 22,5\%. Cinco pacientes (12,5\%) requirieron ATC. Conclusión Aunque se categorizan dentro de los patrones simples, las fracturas de pared posterior son lesiones complejas. La tomografía axial computada preoperatoria es esencial para determinar lesiones intra-articulares dada su alta frecuencia y también permite realizar un buen plan preoperatorio.
\end{abstract}

received

May 16, 2018

accepted

April 18, 2020
DOI https://doi.org/

10.1055/s-0040-1712938. ISSN 0716-4548.
Copyright $\odot 2020$ by Thieme Revinter

Publicações Ltda, Rio de Janeiro, Brazil
License terms

c) $9 \ominus \$$ 
Abstract

\section{Keywords}

- Acetabular fracture

- posterior wall

- reduction and osteosynthesis
Objective Evaluate radiologic results in short and medium-term in surgically treated patients with acetabular posterior wall fracture. Describe fracture morphology, the presence of associated lesions and the necessity of total hip arthroplasty (THA) during the evolution.

Materials and methods A retrospective-descriptive study design of 40 patients treated during July 2012 and July 2017 for acetabular posterior wall fracture. 37 men and 3 women, 41 years old mean age. The mean follow-up was 17.7 months. Accident mechanism and presence of associated lesions were registered, as well as the number of fragments of the fracture, the presence of marginal impaction, femoral head compromise, intra-articular fragments and postoperatory complications. Wilcoxon test was used for the statistical analysis.

Results Every case resulted due to a high energy accident. 70\% of patients had posterior luxation of the hip. $70 \%$ of patients presented 2 to 5 fragments. $47.5 \%$ of patients presented marginal impaction, $37.5 \%$ compromised femoral head and $30 \%$ intra-articular fragments. The most frequent complication was post-traumatic arthrosis, which appeared in $22.5 \%$ of patients. Five patients required THA.

Conclusion Though it is categorized as simple fracture pattern, posterior wall fractures are complex. Preoperatory CT scan is essential to determine intra-articular lesions due to its high frequency and permits to plan an adequate intervention.

\section{Introducción}

Las fracturas de pared posterior corresponden al patrón de fractura más frecuente del acetábulo, llegando a representar entre el $20 \%$ y $30 \%$ del total. ${ }^{1-3}$ Se puede observar compromiso de la pared posterior aislada o asociada a otro patrón de fractura hasta en un tercio de los pacientes. ${ }^{4,5}$

Generalmente, se presentan en la población joven secundario a un accidente de alta energía por lo que es frecuente de encontrar lesiones asociadas a esa lesión.

En general, el tratamiento de ese tipo de lesiones es quirúrgico. Se describe el tratamiento ortopédico sólo para aquellas fracturas en que el fragmento sea pequeño y la estabilidad de la articulación de la cadera se mantenga conservada. $^{4-6}$ Los objetivos del tratamiento quirúrgico son obtener una reducción anatómica y estable, con el fin de recuperar la movilidad y estabilidad de la cadera, y al mismo tiempo disminuir el dolor. Los resultados dependerán de una serie de factores, dentro de los que se encuentran los pre quirúrgicos, como son la edad del paciente, el tipo de fractura, antecedente de luxación, compromiso de la cabeza femoral, presencia de fragmentos osteocondrales intra-articulares, impactación marginal y lesiones asociadas. ${ }^{5,7,8}$ En los factores post quirúrgicos se pueden mencionar el tipo de reducción de la fractura obtenida, osteonecrosis de la cabeza femoral, osificaciones heterotópicas y evolución hacia artrosis de cadera. ${ }^{9}$

Los resultados post-operatorios que se describen en la literatura son en general buenos, pero existe un grupo de pacientes que evolucionarán de manera desfavorable pese a obtener una adecuada reducción de la fractura y las complicaciones pueden llegar hasta el 30\%. ${ }^{10}$

El objetivo de este estudio, es evaluar los resultados radiológicos a corto $\mathrm{y}$ mediano plazo de pacientes operados de fractura de pared posterior de acetábulo, así como describir la morfología de la fractura y presencia de lesiones asociadas.

\section{Material y métodos}

Estudio retrospectivo-descriptivo de una serie consecutiva de 40 pacientes operados entre julio de 2012 y julio de 2017 en el mismo centro hospitalario y por el mismo equipo quirúrgico. Se incluyeron pacientes con fractura de acetábulo cuyo compromiso fuera únicamente de la pared posterior, sin patrones asociados. Treinta y siete hombres y tres mujeres con una edad promedio de 41 años (20-54 años) se operaron en ese período y se les realizó una mediana de 17,7 meses (6-66 meses).

Se registró el mecanismo del accidente y la presencia de lesiones asociadas. En todos los casos se contó con tomografía axial computada (TAC) con reconstrucción 3D preoperatoria. Se registraron el número de fragmentos que presentaba la fractura, presencia de impactación marginal, compromiso de la cabeza femoral y presencia de fragmentos intra-articulares (-Figura 1). Se registró el tiempo transcurrido entre el accidente y la cirugía. Para el análisis estadístico se utilizó test de Wilcoxon considerándose estadísticamente significativo un valor de $\mathrm{p} \leq 0,05$.

Para la cirugía se utilizó mesa radio-lúcida (mesa Jackson). Se posicionó al paciente en decúbito lateral con soporte de ambos lados mediante cojín al vacío. La mesa se inclinó ligeramente hacia ventral del paciente. Mediante abordaje Kocher-Langenbeck se accedió a la fractura. Realizamos resección del músculo glúteo menor de acuerdo a lo publicado por Routt y col. ${ }^{11}$ Se retiraron los fragmentos de la fractura de manera transitoria independiente de su 


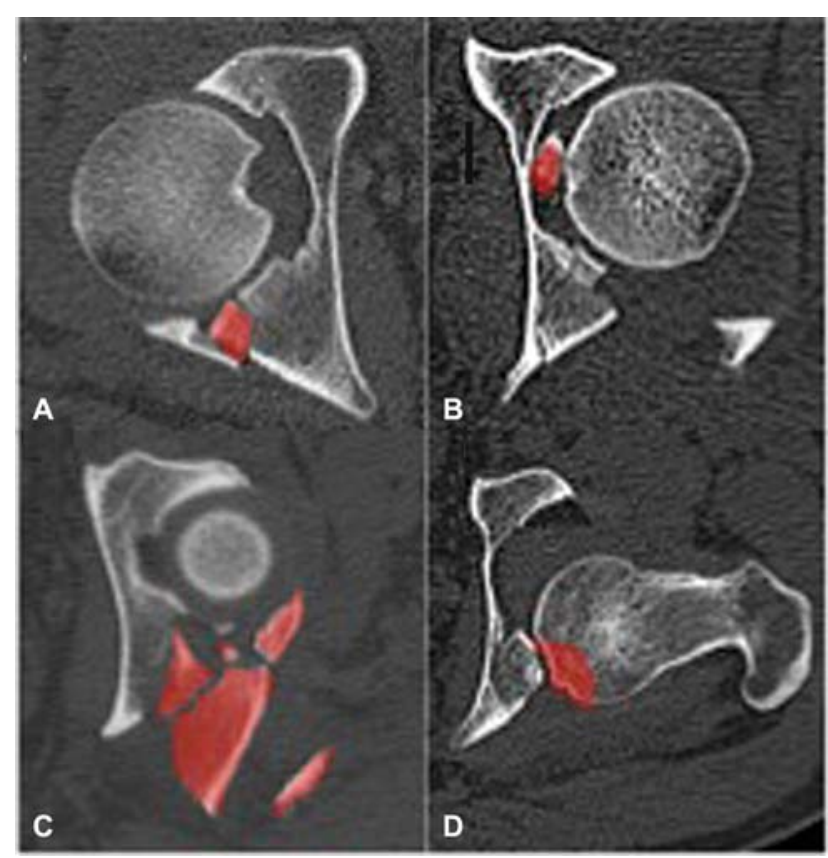

Fig. 1 Cortes axiales de TAC. En rojo se marca: (A) Impactación marginal, (B) Fragmentos de fractura de pared posterior, (C) Fragmento intra-articular, (D) Compromiso de la cabeza femoral.

tamaño. Se realizó aseo profuso de la articulación extrayendo los fragmentos intra-articulares. En caso de impactación marginal, se utilizó injerto de trocánter mayor o aloinjerto. Luego, se volvieron a posicionar los fragmentos de la fractura. Se fijaron de manera transitoria con agujas Kirschner, y luego, dependiendo del tamaño del fragmento, se fijaron con tornillos o spring plate, para finalmente utilizar una placa de $3,5 \mathrm{~mm}$ moldeada. Hubo 2 casos en los que se tuvo que realizar además una osteotomía tipo flip trocantérico con el fin de poder retirar grandes fragmentos intra-articulares que se encontraban localizados hacia anterior y no se podían retirar por posterior (-Figura 2 y 3 ).

En el postoperatorio se indicó fisioterapia con descarga por 6 semanas del lado operado.
Se tomaron radiografías de control en forma mensual hasta la consolidación.

Se registraron las complicaciones postoperatorias. Se consideraron como siendo complicaciones las siguientes: infección, luxación, lesión nervio ciático, artrosis posttraumática, osteonecrosis de la cabeza femoral y osificaciones heterotópicas.

\section{Resultados}

Todos los casos fueron secundarios a un accidente de alta energía ( - Tabla 1), destacando la mayoría de los casos, como secundarios a un accidente automovilístico.

Un $70 \%$ de los pacientes se presentaron de manera asociada con una luxación posterior de cadera. La lesión asociada que mayormente observamos fue la presencia de otras fracturas ( - Tabla 2). Ocho pacientes (20\%) presentaron lesión de nervio ciático al momento del accidente, la cual se pesquisó de manera clínica.

A la evaluación de la TAC, la gran mayoría de los pacientes presentó una fractura entre 2 y 5 fragmentos (70\%). Sólo 6 pacientes presentaron una fractura con fragmento único. Un 47,5\% de los pacientes presentó impactación marginal. Un $37,5 \%$ de los pacientes presentó compromiso de la cabeza femoral en zona de carga. Un 30\% de los pacientes presentó fragmentos intra-articulares (-Gráfico $\mathbf{1}$ ).

El tiempo transcurrido entre el accidente y la cirugía fue en promedio 3,3 días (1-6 días).

La complicación que más frecuente se observó fue la artrosis post-traumática la cual se presentó en 9 pacientes (22,5\%). Dos pacientes (5\%) presentaron osteonecrosis de la cabeza femoral. En un paciente desarrolló osificaciones heterotópicas. En un caso se observó una infección superficial que respondió a un tratamiento antibiótico. Un paciente presentó luxación posterior a la cirugía (- Tabla 3).

Un total de 5 pacientes $(12,5 \%)$ requirió de la realización de una artroplastía total de cadera (ATC) después de 21,8 meses (2-38 meses) promedio de la cirugía inicial. De ellos, 3 fueron debido a artrosis de cadera sintomática y 2 por

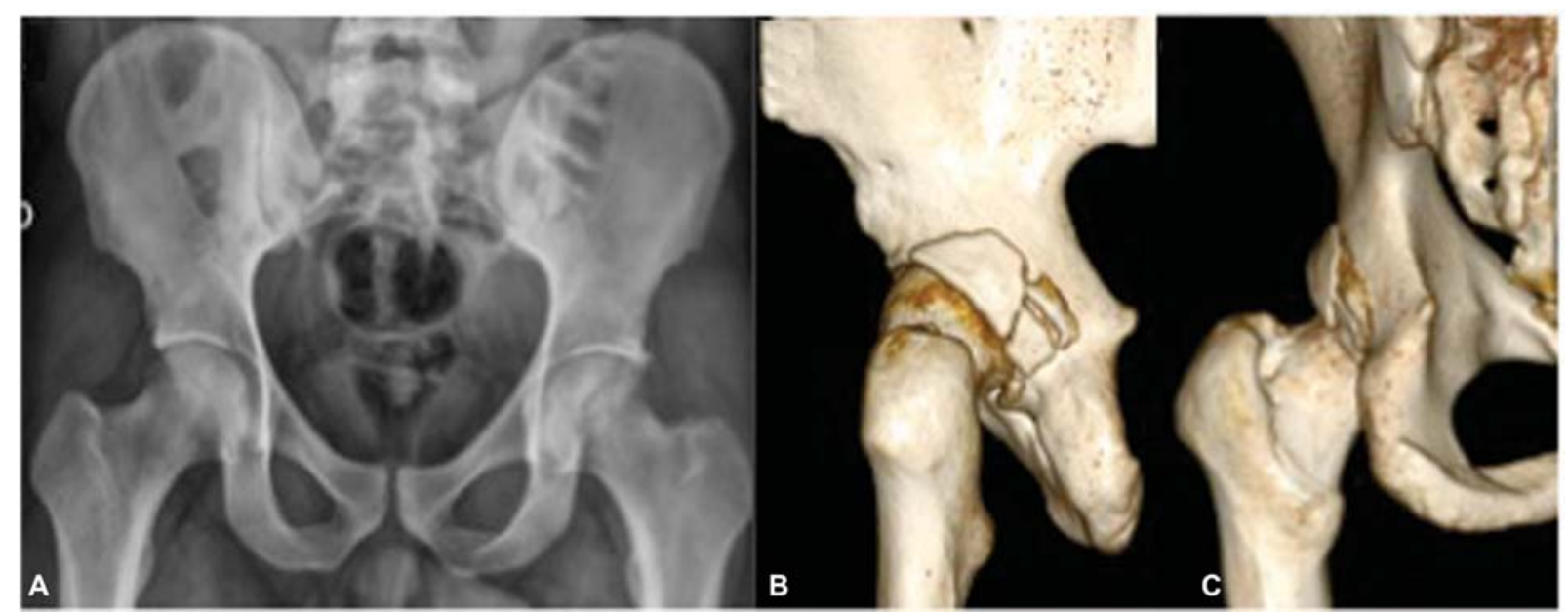

Fig. 2 Caso clínico 1. Paciente de genero masculino de 42 años que sufre accidente motocicleta. (A) Radiografía de pelvis AP en que se muestra compromiso de la pared posterior de acetábulo. B y (C) Reconstrucción 3D de TAC en que muestra fractura de 3 fragmentos. 


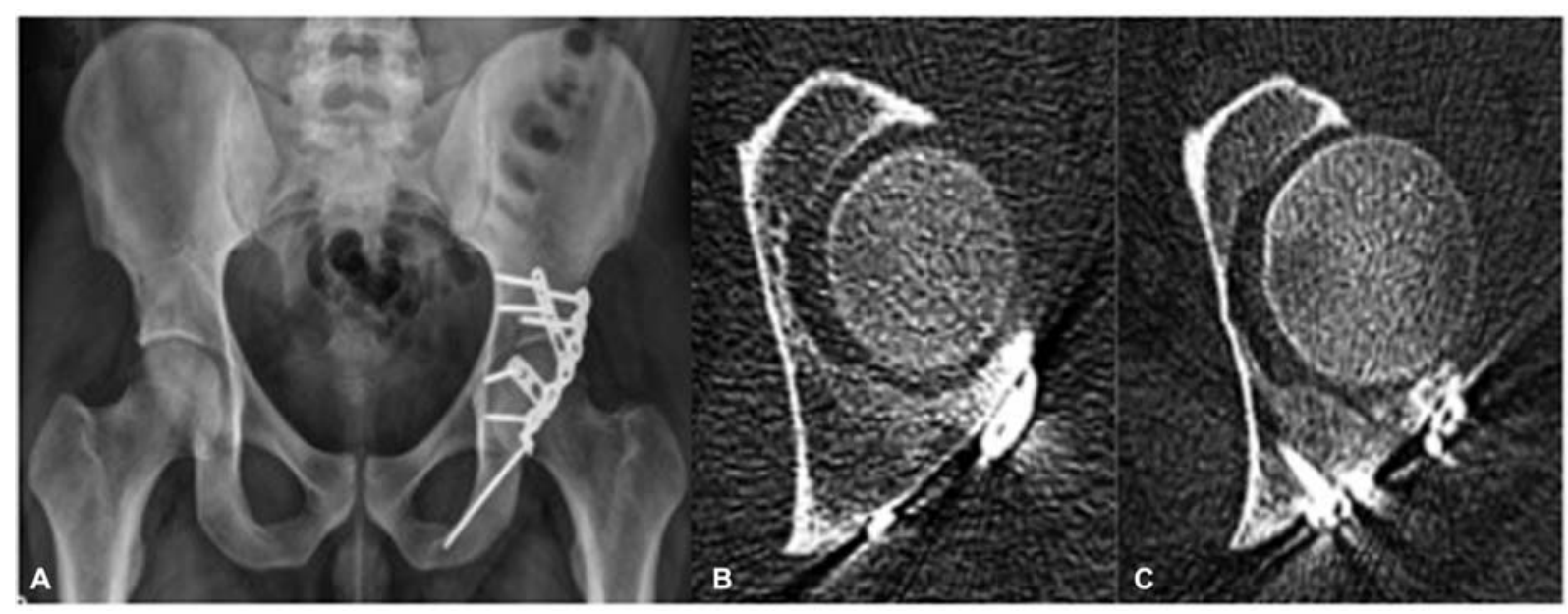

Fig. 3 Imágenes postoperatorias del caso clínico 1. (A) Radiografía de pelvis anterposterior que muestra reducción y osteosíntesis de pared posterior de acetábulo en la que se utilizó placa de 3,5 mm y spring plate. B y (C) Cortes axiales de cadera en la que se muestra congruencia articular.

Tabla 1 Descripción del mecanismo de fractura de los pacientes que presentaron fractura de pared posterior de acetábulo

\begin{tabular}{|l|l|l|}
\hline Mecanismo & Número de casos & Porcentaje \\
\hline Accidente automovilístico & 34 & $84 \%$ \\
\hline Trauma directo & 3 & $8 \%$ \\
\hline Caída de altura & 3 & $8 \%$ \\
\hline
\end{tabular}

Tabla 2 Descripción de las lesiones asociadas que presentaron los pacientes operados

\begin{tabular}{|l|l|l|}
\hline Lesiones asociadas & Número de casos & Porcentaje \\
\hline Otras fracturas & 10 & $25 \%$ \\
\hline Lesión nervio ciático & 8 & $20 \%$ \\
\hline TEC & 3 & $7 \%$ \\
\hline
\end{tabular}

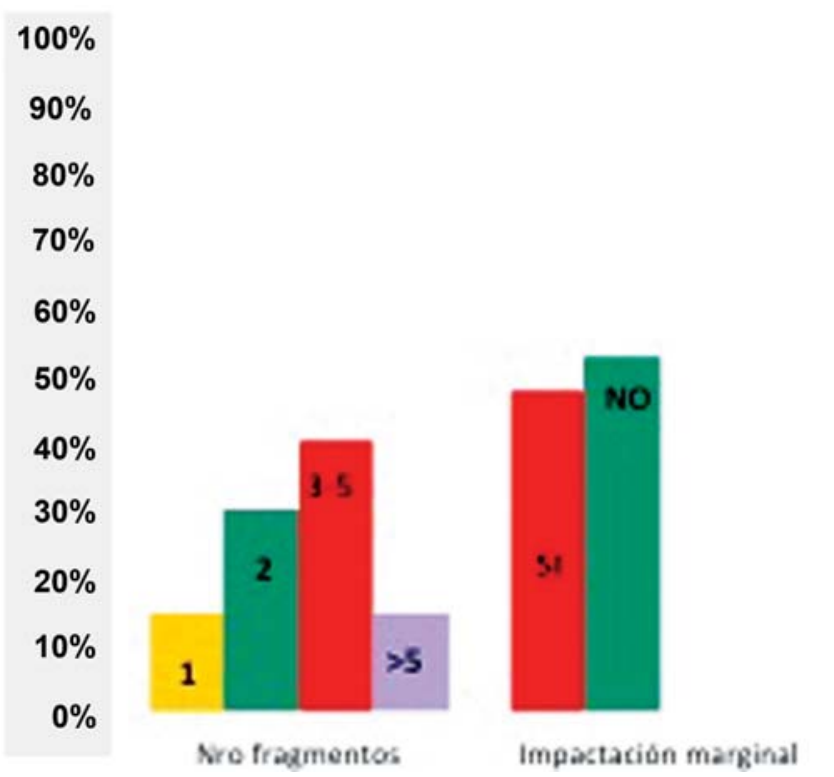

Tabla 3 Complicaciones postoperatorias de los pacientes sometidos a reducción y osteosíntesis de acetábulo

\begin{tabular}{|l|l|l|}
\hline Complicaciones & $\begin{array}{l}\text { Número } \\
\text { de casos }\end{array}$ & Porcentaje \\
\hline Artrosis post-traumática & 9 & $22,5 \%$ \\
\hline Osteonecrosis cabeza femoral & 2 & $5 \%$ \\
\hline Osificaciones heterotópicas & 1 & $2,5 \%$ \\
\hline Luxación de cadera & 1 & $2,5 \%$ \\
\hline Infección partes blandas & 1 & $2,5 \%$ \\
\hline
\end{tabular}

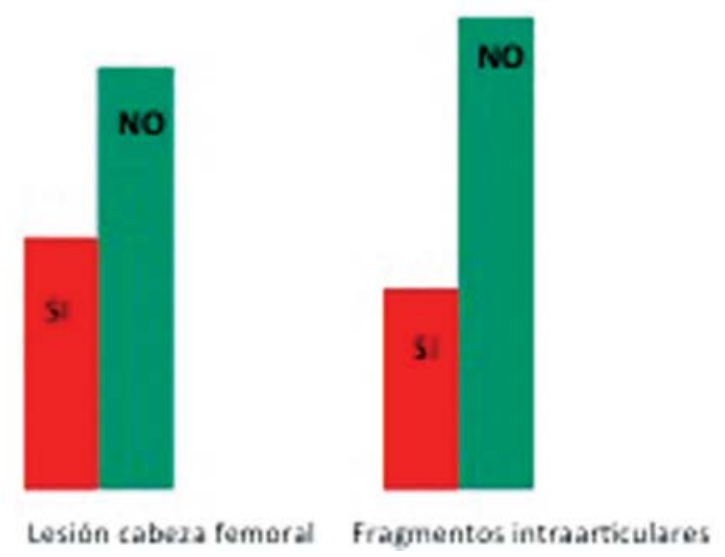

Gráfico 1 Se describen los porcentajes de las características de las fracturas observadas en la tomografía axial computada preoperatoria. 


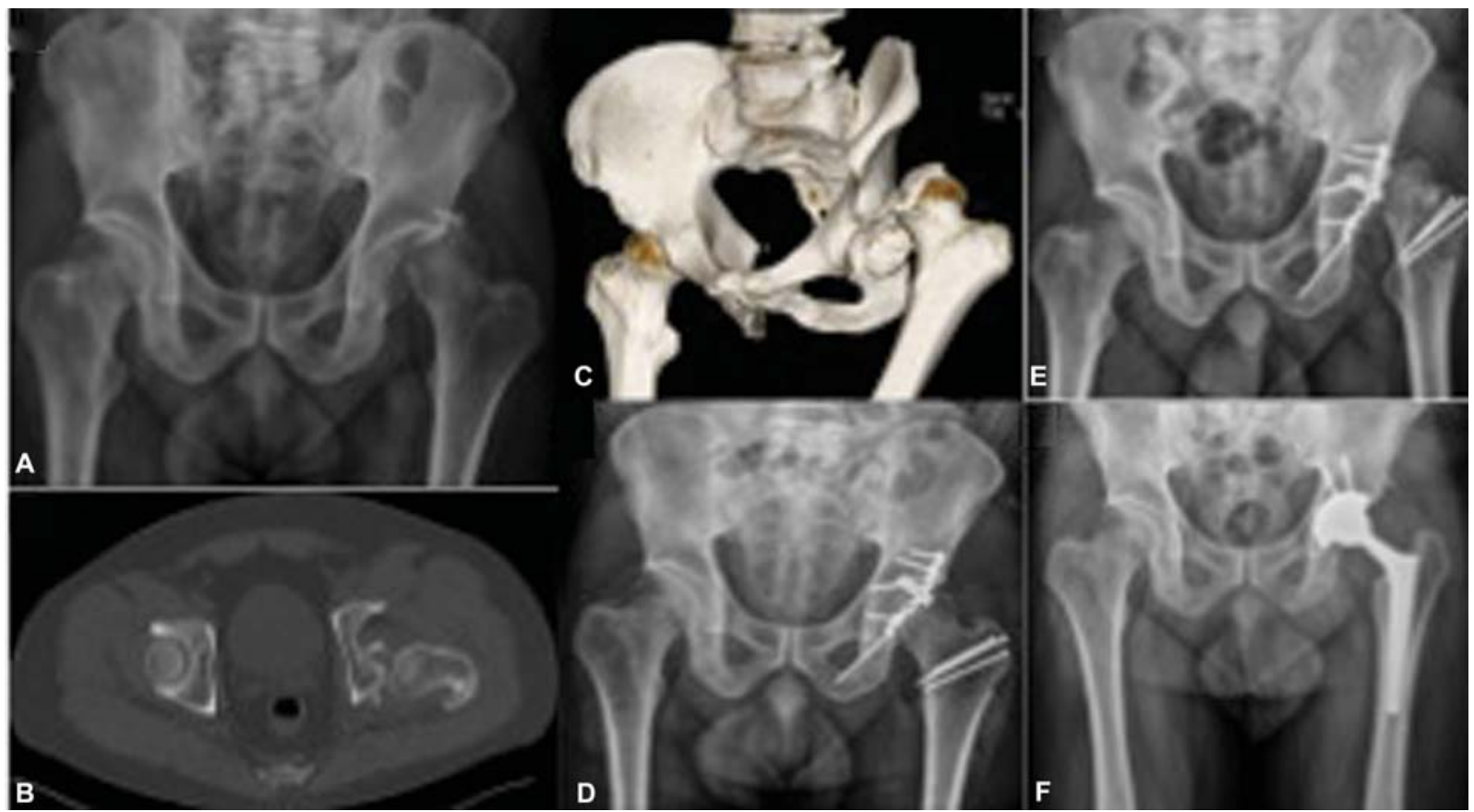

Fig. 4 Caso clínico 2. Paciente de género masculino de 39 años que sufrió accidente automovilístico. (A) Radiografía anteroposterior de pelvis en la que se observa fractura de pared posterior de acetábulo izquierdo asociada a luxación posterior. Además, se puede ver en la cadera derecha déficit de cobertura anterior y posterior de la cabeza femoral lo que habla de una cadera displásica. (B) Corte axial en la que se observan fragmentos intra-articulares. (C) Reconstrucción 3D de la cadera izquierda en la que se observa un gran fragmento de pared posterior. (D) Radiografía anteroposterior de pelvis post-operatoria inmediata en la que se observa uso de placa de 3,5 mm, spring plate y tornillos de 4,5 mm en el trocánter mayor debido a la realización de un flip trocantérico. E: Radiografía anterposterior de pelvis a las 5 semanas de operado en la que se ve luxación posterior de la cadera asociado a osteonecrosis de la cabeza femoral. F: Radiografía anteroposterior de pelvis que muestra ATC a las 8 semanas de la primera cirugía.

Tabla 4 Descripción de los 5 pacientes que requirieron durante su evolución una artroplastia total de cadera

\begin{tabular}{|l|l|l|l|l|l|}
\hline Caso & Luxación & Impactación marginal & Más de 3 fragmentos & Fragmentos intra-articulares & Lesión cabeza femoral \\
\hline 1 & $\mathrm{X}$ & $\mathrm{X}$ & $\mathrm{X}$ & & \\
\hline 2 & & $\mathrm{X}$ & $\mathrm{X}$ & & \\
\hline 3 & $\mathrm{X}$ & & $\mathrm{X}$ & $\mathrm{X}$ & $\mathrm{X}$ \\
\hline 4 & & $\mathrm{X}$ & $\mathrm{X}$ & & \\
\hline 5 & $\mathrm{X}$ & $\mathrm{X}$ & $\mathrm{X}$ & & \\
\hline
\end{tabular}

osteonecrosis de la cabeza femoral. De esos últimos, uno fue por luxación y el otro por dolor ( - Figura 4). En la - Tabla 4 se presentan las características de los 5 pacientes que requirieron una ATC. Tanto la presencia de impactación marginal como la de presentar una fractura conminuta con más de 3 fragmentos mostraron diferencia significativa $(\mathrm{p} \leq 0,05)$ para la ATC.

\section{Discusión}

Las fracturas de pared posterior de acetábulo se consideran muchas veces lesiones de fácil manejo debido al buen acceso a la articulación gracias a un abordaje que así lo permite, el abordaje de Kocher Langenbeck resulta familiar para los cirujanos de cadera, especialmente aquellos que lo utilizan en la realización de ATC. Sin embargo, los resultados postoperatorios muchas veces no son buenos. Eso se debe a las características de las fracturas, en las que la conminución y la impactación marginal juegan un rol clave. ${ }^{12}$ De hecho, tener una fractura de pared posterior con fragmento único es poco frecuente, solo 6 pacientes de nuestra serie. Lo habitual es que los pacientes presenten conminución. Tanto en las series de Letournel ${ }^{3}$ y Matta ${ }^{5}$ se reportan reducciones anatómicas en un 94 y 100\% respectivamente, no obstante, los resultados clínicos fueron buenos sólo en un 68 y 82\% respectivamente. Esa diferencia se explica por la conminución de la fractura, la presencia de impactación marginal, lesión de la cabeza femoral y la osteonecrosis. Es por ese motivo que se pueden encontrar en la literatura resultados diferentes para ese tipo de fracturas, ya que dependerá de la presencia o no de esos factores. Es así como se pueden encontrar resultados buenos y excelentes sobre el $85 \%^{13}$ y otros con pérdida de la reducción sobre el $30 \%$ al año después de la cirugía, ${ }^{14}$ por lo que la calidad de la reducción también juega un rol importante. 
Debido a que esas lesiones se producen secundarias a un accidente de alta energía, es frecuente encontrar otras lesiones. La lesión asociada que más frecuentemente observamos en nuestra serie fue la luxación. En general, esa lesión por sí sola no marca el pronóstico de la fractura en sí ya que siempre se reducen de manera aguda. Diferente es si asociado a la luxación se presenta compromiso del nervio ciático. Es esa lesión la que marcará de mayor manera la evolución del paciente independiente que la reducción de la fractura sea anatómica. ${ }^{15}$ En nuestra serie se observó compromiso del nervio ciático en un $20 \%$ de los pacientes al momento de presentar la fractura.

Magu y col. ${ }^{16}$ refieren que pacientes con fracturas asociadas en extremidades inferiores presentan peores resultados. De nuestra serie, el $25 \%$ de los pacientes presentaron fracturas asociadas. A nuestro entender, en fracturas que no involucren la cadera, sólo se retrasa la carga del paciente. Si se presentan lesiones en la cadera se incrementa más el riesgo de desarrollo de artrosis post-traumática y osteonecrosis.

En la evaluación imagenológica mediante TAC, la mayoría de los pacientes presentó una fractura conminuta. Ese factor es mencionado en las diferentes series como de mal pronóstico para el tratamiento de esas fracturas. Es junto a la presencia de impactación marginal y el estado del cartílago nativo los que marcarán de mayor manera la evolución de esas lesiones después de la cirugía. La impactación marginal se reporta en alrededor del $30 \%$ de los pacientes. ${ }^{17,18}$ Para el tratamiento de esa lesión en particular se plantea rellenar la cavidad con el fin de recuperar la relación de la cabeza femoral dentro del acetábulo. Para ello, se debe primero identificar la impactación, luego levantar el fragmento y rotarlo a su posición natural. Una vez realizado ese procedimiento, se debe rellenar el defecto remanente. Para ello, se plantea el uso de auto injerto ya sea de cresta ilíaca o del trocánter mayor. En nuestra serie un $45,7 \%$ de los pacientes presentó impactación. ${ }^{19}$ Para su manejo, nosotros preferimos rellenar la cavidad con injerto proveniente del trocánter mayor. Consideramos que se logra obtener la cantidad suficiente de hueso con una mínima morbilidad asociada. En pacientes con grandes defectos también podemos usar aloinjerto.

Cuando existe compromiso de la cabeza femoral, se considera también de mal pronóstico. En nuestra serie se presentó en un 37,5\% de los casos. Diferentes series muestran que en el manejo agudo no hay tratamiento enfocado para resolverlo, salvo tener la certeza que se extraen todos los fragmentos articulares libres que puedan estar presentes. Los fragmentos intra-articulares que en general se encuentran, provienen tanto de la cabeza femoral como de la fractura. Es crucial contarlos previo a la cirugía y evitar que la cadera se vuelva a luxar, ya que ello puede cambiar en número de fragmentos intra-articulares. En caso de persistencia de fragmentos en el postoperatorio, esos se podrían extraer mediante artroscopia.

En nuestra serie, los pacientes se operaron en promedio a los 3,3 días. Diferentes estudios plantean que el momento de la cirugía no marca el pronóstico si es antes de las 3 semanas. Lo que está demostrado es la demora en la reducción de la cadera luxada. ${ }^{20}$
Un 12,5\% de nuestra serie requirió la realización de una ATC. La causa fue en 3 casos artrosis sintomática y 2 osteonecrosis. De estos pacientes todos presentaron una fractura conminuta y un $80 \%$ impactación marginal. Magu y col. ${ }^{16}$ en su serie retrospectiva de 25 pacientes, reportan un $12 \%$ de ATC después de cirugía de pared posterior de acétabulo a los 12 años de seguimiento. Pascarella y col. ${ }^{21}$ reportan en su serie de 121 pacientes un $16,5 \%$ de ATC al final del seguimiento que fue en promedio a los 53 meses. Refieren que el factor predominante en ese grupo, fue la lesión inicial de la cabeza femoral. Misitonis y col. ${ }^{10}$ en una serie retrospectiva de 17 pacientes seguida por 15 años, reportan un 5,3\% de ATC al final del seguimiento.

\section{Conclusión}

Las fracturas de pared posterior son lesiones complejas a pesar de su denominación de simple o elemental al clasificarlas. La TAC preoperatoria es esencial para determinar lesiones intrarticulares dado su alta frecuencia y permite realizar un buen plan preoperatorio.

\section{Conflictos de Intereses}

Los autores declaran no tener ningún conflicto de intereses.

\section{Bibliografía}

1 Aho AJ, Isberg UK, Katevuo VK. Acetabular posterior wall fracture. 38 cases followed for 5 years. Acta Orthop Scand 1986;57(02): 101-105

2 Baumgaertner MR. Fractures of the posterior wall of the acetabulum. J Am Acad Orthop Surg 1999;7(01):54-65

3 Letournel E, Judet R. Fractures of the Acetabulum. 2nd ed. New York, NY: Springer; 1993

4 Grimshaw CS, Moed BR. Outcomes of posterior wall fractures of the acetabulum treated nonoperatively after diagnostic screening with dynamic stress examination under anesthesia. J Bone Joint Surg Am 2010;92(17):2792-2800

5 Matta JM. Fractures of the acetabulum: accuracy of reduction and clinical results in patients managed operatively within three weeks after the injury. J Bone Joint Surg Am 1996;78(11):16321645

6 Moed BR, Dickson KF, Kregor PJ, Reilly MC, Vrahas MS. The surgical treatment of acetabular fractures. Instr Course Lect 2010;59: 481-501

7 Mears DC, Velyvis JH. Primary total hip arthroplasty after acetabular fracture. Instr Course Lect 2001;50:335-354

8 Moed BR, Carr SE, Gruson KI, Watson JT, Craig JG. Computed tomographic assessment of fractures of the posterior wall of the acetabulum after operative treatment. J Bone Joint Surg Am 2003; 85(03):512-522

9 Siebenrock KA, Gautier E, Ziran BH, Ganz R. Trochanteric flip osteotomy for cranial extension and muscle protection in acetabular fracture fixation using a Kocher-Langenbeck approach. J Orthop Trauma 1998;12(06):387-391

10 Mitsionis GI, Lykissas MG, Motsis E, et al. Surgical management of posterior hip dislocations associated with posterior wall acetabular fracture: a study with a minimum follow-up of 15 years. J Orthop Trauma 2012;26(08):460-465

11 Rath EM, Russell GV Jr, Washington WJ, Routt ML Jr. Gluteus minimus necrotic muscle debridement diminishes heterotopic ossification after acetabular fracture fixation. Injury 2002;33(09): 751-756

12 Ebraheim NA, Patil V, Liu J, Sanford CG Jr, Haman SP. Reconstruction of comminuted posterior wall fractures using 
the buttress technique: a review of 32 fractures. Int Orthop 2007; 31(05):671-675

13 Moed BR, WillsonCarr SE, Watson JT. Results of operative treatment of fractures of the posterior wall of the acetabulum. J Bone Joint Surg Am 2002;84(05):752-758

14 Saterbak AM, Marsh JL, Nepola JV, Brandser EA, Turbett T. Clinical failure after posterior wall acetabular fractures: the influence of initial fracture patterns. J Orthop Trauma 2000;14 (04):230-237

15 Bogdan Y, Tornetta P III, Jones C, et al. Neurologic injury in operatively treated acetabular fractures. J Orthop Trauma 2015; 29(10):475-478

16 Magu NK, Gogna P, Singh A, et al. Long term results after surgical management of posterior wall acetabular fractures. J Orthop Traumatol 2014;15(03):173-179
17 Alonso JE, Volgas DA, Giordano V, Stannard JP. A review of the treatment of hip dislocations associated with acetabular fractures. Clin Orthop Relat Res 2000;(377):32-43

18 Giannoudis PV, Kanakaris NK, Delli Sante E, Morell DJ, Stengel D, Prevezas N. Acetabular fractures with marginal impaction: midterm results. Bone Joint J 2013;95-B(02):230-238

19 Martins e Souza P, Giordano V, Goldsztajn F, Siciliano AA, Grizendi JA, Dias MV. Marginal impaction in posterior wall fractures of the acetabulum. AJR Am J Roentgenol 2015;204(04):W470-4

20 Cahueque M, Martínez M, Cobar A, Bregni M. Early reduction of acetabular fractures decreases the risk of post-traumatic hip osteoarthritis? J Clin Orthop Trauma 2017;8(04):320-326

21 Pascarella R, Cerbasi S, Politano R, et al. Surgical results and factors influencing outcome in patients with posterior wall acetabular fracture. Injury 2017;48(08):1819-1824 